\title{
Agenesis of the Gallbladder: A Phantom Menace
}

\author{
Kara Cherisse Nadeau ${ }^{1}$, Henry Y. Jiang ${ }^{2}$, Rachelle Paradis ${ }^{2}$ \\ ${ }^{1}$ University of Ottawa, Faculty of Medicine, Ontario, Canada \\ ${ }^{2}$ Department of General Surgery, Northern Ontario School of Medicine, Sudbury, Ontario, Canada
}

\section{ABSTRACT}

Objective: Gallbladder agenesis (GBA) is a rare congenital disorder with an estimated incidence of about $0.06 \%$. Despite the absence of a gallbladder, these patients may present with symptoms mimicking biliary colic or cholecystitis. Ultrasound findings and liver function tests are often misleading. Some of these patients undergo laparoscopy without successful identification of gallbladder and paradoxically report symptom relief.

Case: We present a case of GBA in a 54 year-old female, who presented with right-sided abdominal pain. The clinical history and examination were consistent with biliary colic. Initial investigations, including liver function tests, upper endoscopy and ultrasound did not demonstrate upper gastrointestinal pathology and did not clearly identify a gallbladder. Subsequent HIDA scan and CT of the abdomen did not visualize a gallbladder. An MRCP confirmed gallbladder agenesis. The patient was managed conservatively and was symptom free on discharge and follow-up.

Discussion: We wish to highlight four learning points: 1. Patients with gallbladder agenesis often present with biliary symptoms. 2. Ultrasound and CT of the liver may not always identify this anomaly. 3. MRCP is the gold standard for making a diagnosis of gallbladder agenesis. 4. Surgeons must have a high index of suspicion of GBA when the gallbladder is poorly visualized or not identified on ultrasound.

RÉSUMÉ

Objective: Agénésie de la vésicule biliaire (AVB) est un désordre congénital rare avec une incidence d'environ $0.06 \%$. Malgré l'absence d'une vésicule biliaire, ces patients peuvent se présenter avec des symptômes qui imitent une colique biliaire ou une cholécystite. Les trouvailles à l'echographie et les tests de la fonction hépatique sont souvent trompeurs. Quelques patients subissent une laparoscopie, sans identification de la vésicule biliaire, mais rapportent une amélioration de symptômes paradoxalement.

Cas: Nous présentons un cas de AVB chez une femme de 54 ans, qui se présentait avec une douleur abdominale droite. L'historique et l'examen physique étaient concordants avec une colique biliaire. Les investigations initiales, y inclut les tests de la fonction hépatique, l'endoscopie haute et l'echographie, n'ont pas démontré une pathologie gastro-intestinale supérieure et n'ont pas identifié clairement une vésicule biliaire. Ni une scintigraphie HIDA ni une TDM abdominale n'ont visualisé une vésicule biliaire. Une CPRM a confirmé l'agénésie de la vésicule biliaire. La patiente a été gérée de façon conservatrice et a été asymptomatique lors de son congé et suivie.

Discussion: Nous voulons surligner quatre points d'enseignement : 1. Les patients avec l'agénésie de la vésicule biliaire se présentent souvent avec des symptômes biliaires. 2. L'echographie et la TDM du foie ne trouvent pas toujours cette anomalie. 3. CPRM est le test de référence pour le diagnostic de l'agénésie de la vésicule biliaire. 4. Les chirurgiens doivent avoir une haute suspicion de l'AVB quand la vésicule biliaire n'est pas facilement visualisée ou identifiée à l'ultrason.

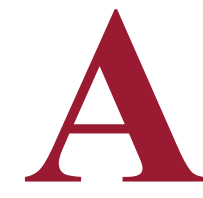

54 year-old female presents to the Emergency Department with right-sided abdominal pain radiating to her back. The pain has been intermittent for several months, is aggravated by meals and is associated with nausea without vomiting or diarrhea. There has been no history of jaundice. Her past surgical history is significant for a Roux-en-Y gastric bypass surgery, during which there was a planned concurrent cholecystectomy but the gallbladder could not be identified. She is known to have mild dumping syndrome since her bypass.
On examination, her vital signs are normal. She is tender in the right abdomen and has a positive Murphy's sign. All laboratory tests, including complete blood count, liver function tests, bilirubin and lipase are normal. An abdominal ultrasound is performed but her gallbladder cannot be visualized (Figure 1). Upper endoscopy is performed to rule out peptic ulcer disease and is normal. Because of the clinical suspicion that her symptoms were biliary in nature, a HIDA scan is done (Figure 2) which demonstrates no filling of the gallbladder. A CT scan of the abdomen shows a wedge-shaped area of

Keywords: Gallbaldder agenesis; Biliary surgery; Laproscopy 


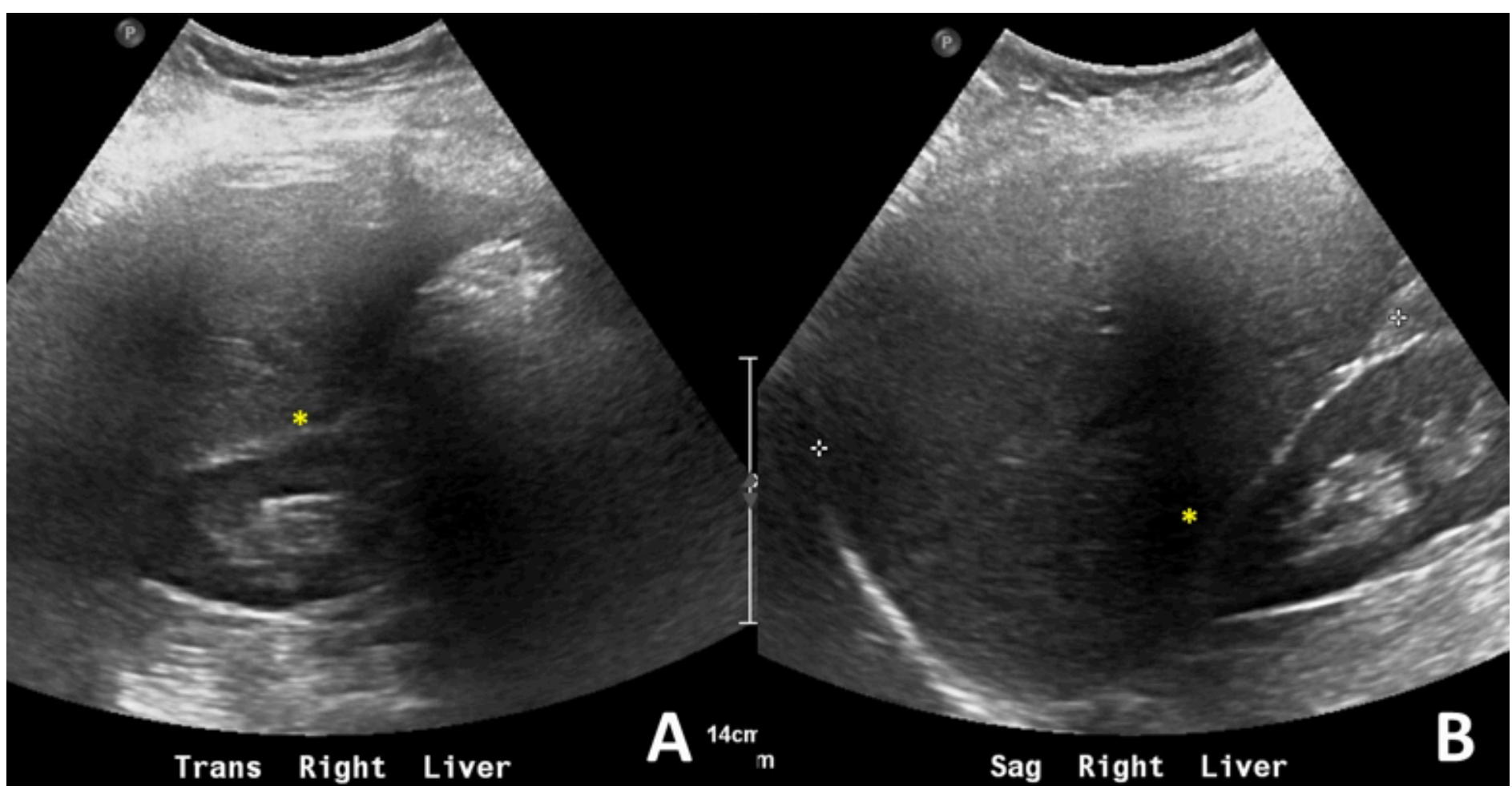

Figure 1. Ultrasound images from our patient with gallbladder agenesis. Stars $\left(^{*}\right)$ are located at the interface between the liver and right kidney, and the lack of a fluid-filled sac in the subhepatic space represents the absence of a gallbladder. (A) Sagittal view of the liver. (B) Transverse view of the liver.

hypoattenuation at the expected location of the gallbladder fossa without appreciation of a gallbladder (Figure 3). Having failed to obtain a definitive diagnosis, a MRCP is done which confirms absence of a gallbladder consistent with a diagnosis of gallbladder agenesis (Figure 4). The patient is managed nonoperatively and improves.

\section{DISCUSSION}

\section{Epidemiology}

Gallbladder agenesis is an anomaly that results from the failure of budding of the gallbladder and cystic duct from the common bile duct during the fourth and fifth weeks of embryogenesis (1). The first case reports were published in 1701 by Lemery and Bergman (2). It is exceedingly rare with an estimated incidence of about $10-65$ per 100,000 in clinical practice, predominating in middle aged women (2). In autopsy series, the incidence is estimated to be higher at 90 per 100,000 with both sexes equally affected (2). It has been suggested that there may be a familial association since it has been noted to occur in familial clusters, but no specific genotypic predisposition has been identified (1).
Presentation

Based on clinical presentation and physical findings, GBA has been classified into three types: asymptomatic, symptomatic, and associated with other congenital defects. Asymptomatic GBA is identified incidentally on imaging studies or at autopsy. Patients with symptomatic GBA represent over 50\% of cases. They often present, interestingly, with biliary colic, dyspepsia or jaundice $(2,3)$. It is in this group that diagnosis becomes a challenge. Patients are often subjected to a surgical intervention with a presumed diagnosis of cholelithiasis. Hence, in a number of cases of symptomatic GBA, the diagnosis is made intraoperatively (4). Congenital defects often associated with GBA include cardiac, gastrointestinal, and genitourinary abnormalities such as duodenal atresia, intestinal malrotation, pancreas divisum, imperforate anus, hypoplasia of the right hepatic lobe, duplication cysts of the hepatic flexure, ventricular septal defect, renal agenesis, undescended testes, and syndactyly $(1,3)$. It can also be associated with genetic syndromes such as trisomy 18 (1).

It is theorized that symptoms in symptomatic GBA may arise from sphincter of Oddi dysfunction, since sphincter spasms may mimic the symptoms of biliary colic $(3,4)$. Interestingly, the 
CASE REPORT

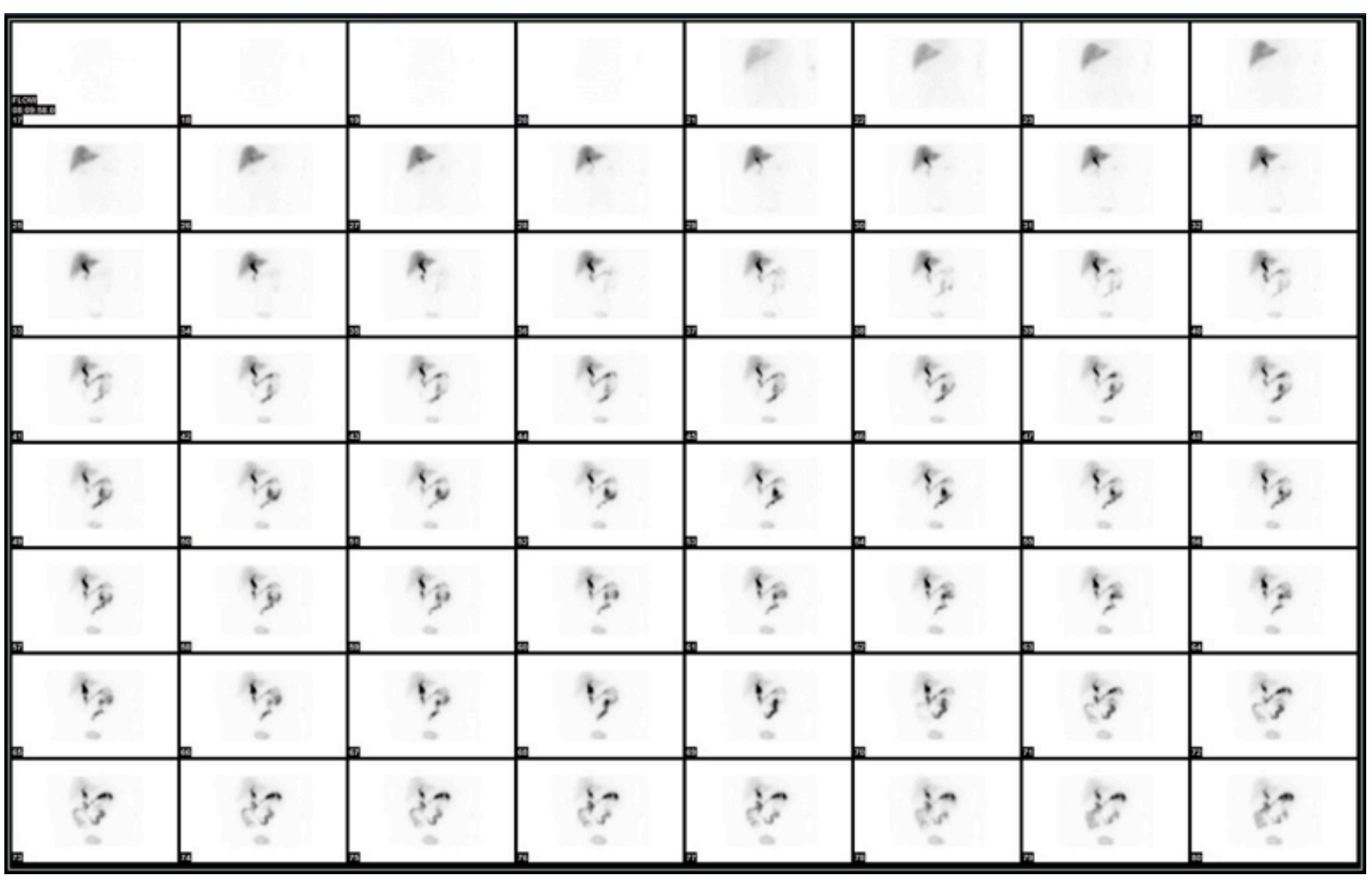

Figure 2. HIDA scan imaging of gallbladder agenesis

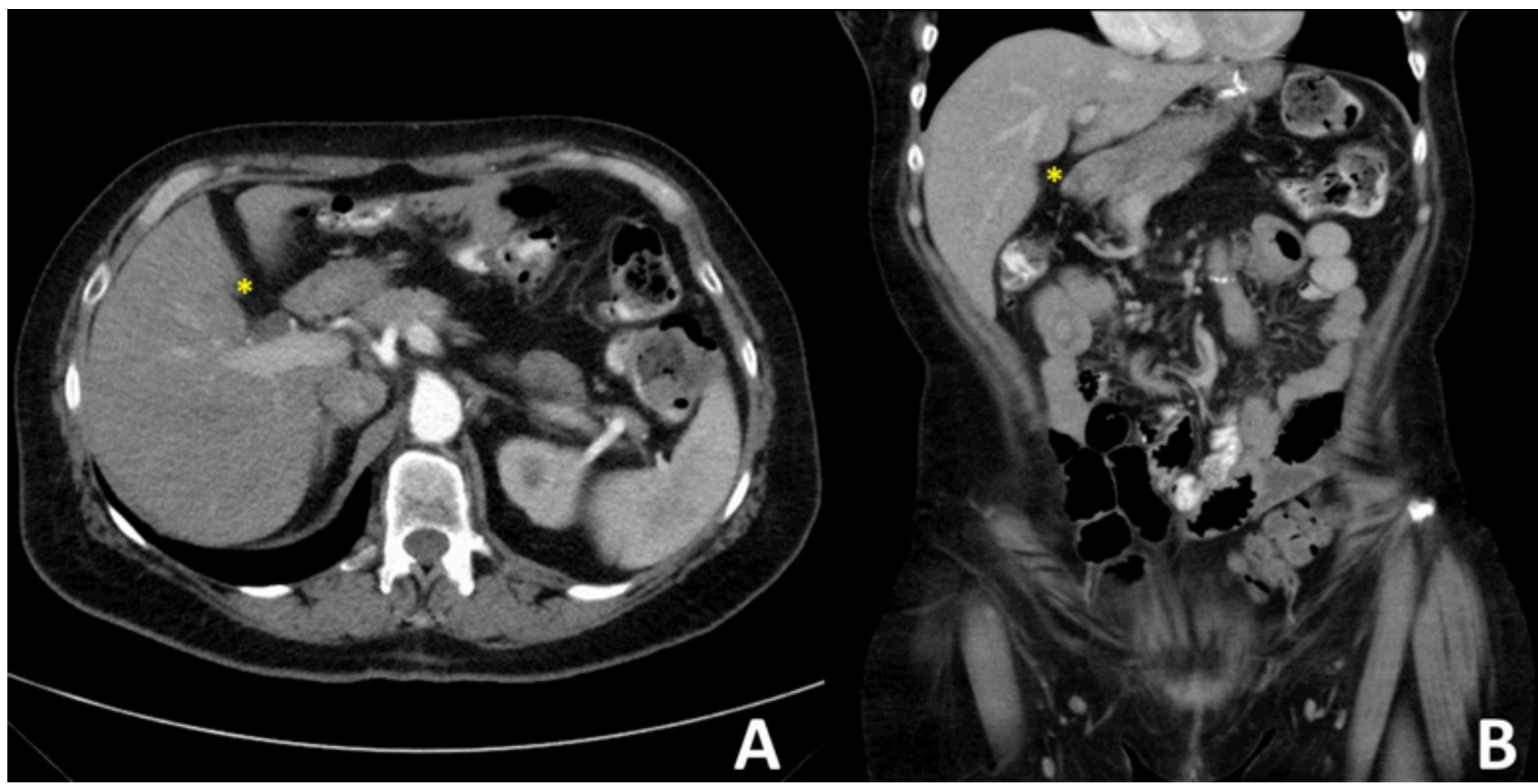

Figure 3. Imaging by CT scan in the patient with gallbladder agenesis. Stars $\left(^{*}\right)$ are found in the fossa on both views to highlight the absence of a gallbladder in that location. (A) Axial views. (B) Coronal views. 


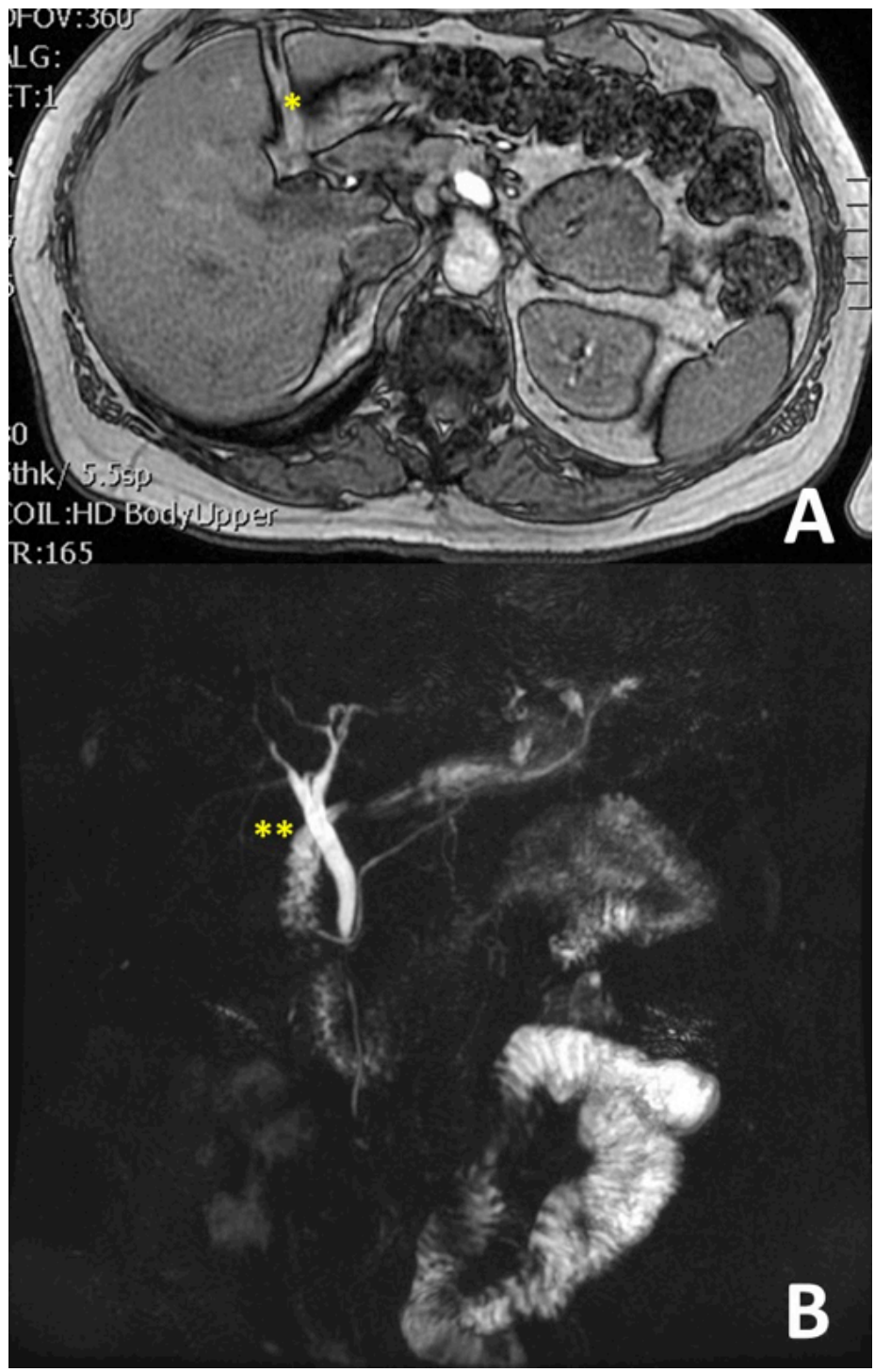

Figure 4. MRCP imaging demonstrating gallbladder agenesis. (A) T1 axial view. Star $\left(^{*}\right)$ represents the lack of gallbladder in the normal gallbladder fossa. (B) MRCP 22 reconstruction of the biliary tree, which consists of the common bile duct and left and right hepatic ducts. There is no evidence of a cystic duct or gallbladder. Stars $\left({ }^{* *}\right)$ present at the expected location of the cystic duct and gallbladder.

frequency of primary stones in the common bile duct in patients with GBA (23\%) is much higher than the post-cholecystectomy rate (5\%). This finding may support the theory that a sphincter of Oddi dysfunction leads to both symptoms and stasis in patients with GBA, and thus to choledocholithiasis $(3,4)$.

Although the morbidity and mortality associated with a nontherapeutic laparoscopic cholecystectomy is low, the risk of complications during an extensive search for an ectopic gallbladder is increased (5). The risks of iatrogenic injury are increased when normal anatomical landmarks are missing and traction on the gallbladder to dissect the triangle of Callot is not feasible (5). There are cases of undiagnosed GBA that have undergone extensive laparoscopic exploration searching for an ectopic gallbladder, with occasional conversion to laparotomy, addition of intraoperative cholangiography through the common bile duct, intraoperative ultrasound, and eventual abandonment of the procedure for further post-operative imaging (5). The option of terminating the procedure and pursuing additional imaging is favored in the literature in order to prevent iatrogenic injury when accurate imaging modalities are now widely available (6). Surgeons need to be aware of this anomaly and maintain a high index of suspicion for GBA when initial investigations do not clearly demonstrate the presence of a gallbladder or biliary disease in order to avoid these intraoperative dilemmas.

In 1967, Frey et al. published that "The preoperative diagnosis of agenesis of the gallbladder is impossible" and thus only a thorough dissection of the entire biliary tract during laparotomy and intraoperative cholangiograhy could confirm the diagnosis (7). However, in the 21st century, imaging modalities have significantly improved and can provide an accurate diagnosis when there is a high index of suspicion for GBA (7). Malde proposes an algorithm (Figure 5) in which radiological investigations are used preoperatively based on available modalities, including MRCP, CT, ERCP, and EUS (6).

Investigations

Laboratory findings for symptomatic GBA are often unremarkable. In patients with choledocholithiasis, there may be corresponding transient elevations in liver function tests and lipase.

Ultrasound often provides the first clues to GBA. When the gallbladder is not clearly visualized on ultrasound, additional investigations should be directed at excluding this diagnosis. In many cases, GBA is diagnosed only intraoperatively, often after the US reports the presence of a contracted or fibrotic gallbladder (8). Many factors, such as body habitus or presence of bowel gas, can obscure visualization of the gallbladder and may lead to confusion $(2,3)$. In cases where the WES triad (visualized gallbladder wall, echo from gallstone, and acoustic shadow) or the double-arc shadow are not clearly seen, both clinicians and radiologists must consider GBA in their differential diagnosis $(4,8)$. 


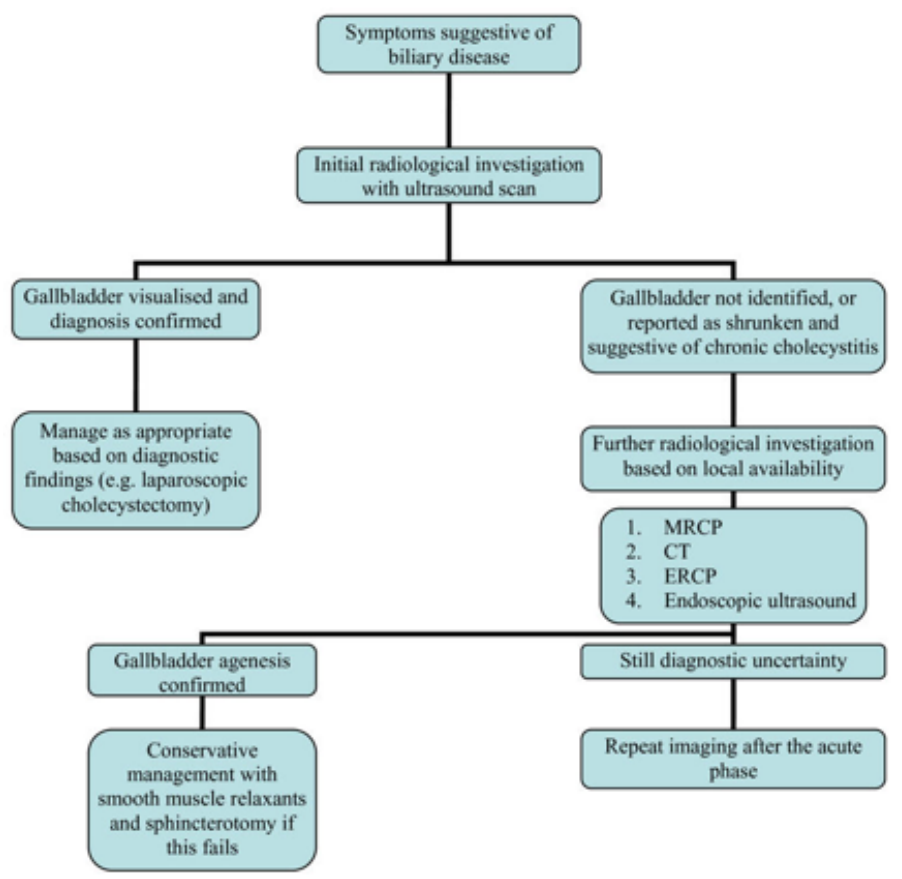

MRCP= magnetic resonance cholangiopancreatography $E R C P=$ endoscopic retrograde cholangiopancreatography

Figure 5. Decisional algorithm in suspected gallbladder agenesis proposed by Malde (6)

HIDA scan is not helpful in differentiating between a diagnosis of acute cholecystitis and GBA as the gallbladder is not filled in both situations. ERCP may also be misleading as non-filling of the gallbladder could be attributed to an obstructed cystic duct or technical errors (8). Most individuals consider GBA to be the least likely diagnosis (7).

MRCP is the modality of choice for diagnosing GBA. With T1 and $\mathrm{T} 2$ phases, the presence or absence of the gallbladder is easily identified. In addition, it also provides clinicians with information regarding any other abnormalities in the biliary tree, including choledocholithiasis, abnormal/variant anatomy, as well as ectopic gallbladder $(3,5,8)$. In cases when MRCP is not readily available, CT scan may also be used $(6,8)$.

\section{Treatment}

There is no consensus as to how to approach this rare condition. Based on case reports, $98 \%$ of symptomatic patients who undergo laparoscopy for possible cholecystectomy have symptom resolution with nontherapeutic laparoscopic cholecystectomy $(2,3)$. One cannot help but ponder on the pathophysiological mystery of this disease, which presents with biliary colic when no gallbladder exists, and resolves when no gallbladder is removed. It is theorized that symptomatic GBA is due to sphincter of Oddi dysfunction, which explains the intermittent nature of the biliary colic in these patients, but not the resolution with nontherapeutic procedures. Some physicians have used hyoscyamine, a levoratory isomer of atropine that acts as a smooth muscle relaxant with success (2). In instances of choledocholithiasis in patients with GBA, ERCP with sphincterotomy and stone extraction is often successful (5).

In our case, after excluding peptic ulcer disease and choledocholithiasis, and confirming the diagnosis of GBA, the patient was managed conservatively with oral analgesia. Her symptoms improved and she was discharged home in stable condition, avoiding an unnecessary operation.

\section{CONCLUSION}

GBA is a rare disease that poses a diagnostic challenge for surgeons. GBA needs to be considered in the differential diagnosis of patients presenting with biliary colic and inconclusive initial ultrasound findings. Appropriate imaging studies such as MRCP are particularly helpful to confirm the diagnosis. Conservative management is often effective and avoids an unnecessary surgery with its associated potential morbidities.

\section{REFERENCES}

1. Yoldas O, Yazici P, Ozsan I, Karabuga T, Alpdogan O, Sahin E, Aydin U. Coexistence of Gallbladder Agenesis and Cholangiocarcinoma: Report of a Case. J Gastrointest Surg. 2014;18(7):1373-1376.

2. Kasi PM, Ramirez R, Rogal SS, Littleton K, Fasanella KE. Gallbladder Agenesis. Case Rep in Gastroenterol. 2011;5(3):654-662.

3. Yener O, Buldanli MZ, Eksioglu H, Leblebici M, Alimoglu O. Agenesis of the Gallbladder Diagnosed by Magnetic Resonance Cholangiography: Report of a Case and Review of the Literature. Prague Med Rep. 2015;116(1):52-56.

4. Tagliaferri E, Bergmann H, Hammans S, Shiraz A, Stuber E, Seidlmayer C. Agenesis of the Gallbladder: Role of Clinical Suspicion and Magnetic Resonance to Avoid Unnecessary Surgery. Case Rep Gastroenterol. 2017;10(3):819-825.

5. Peloponissios N, Gillet M, Cavin R, Halkic N. Agenesis of the gallbladder: A dangerously misdiagnosed malformation. World J Gastroenterol. 2005;11(39):6228-6231.

6. Malde S. Gallbladder agenesis diagnosed intra-operatively: a case report. J Med Case Rep. 2010;4:285.

7. Frey C, Bizer L, Ernst C. Agenesis of the gallbladder. Am J Surg. 1967;114(6):917-926.

8. Mittal A, Singla S, Singal R, Mehta V. Gallbladder agenesis with common bile duct stone - A rare case with a brief review of the literature. Turk J Gastroenterol. 2011;22(2):216-218. 